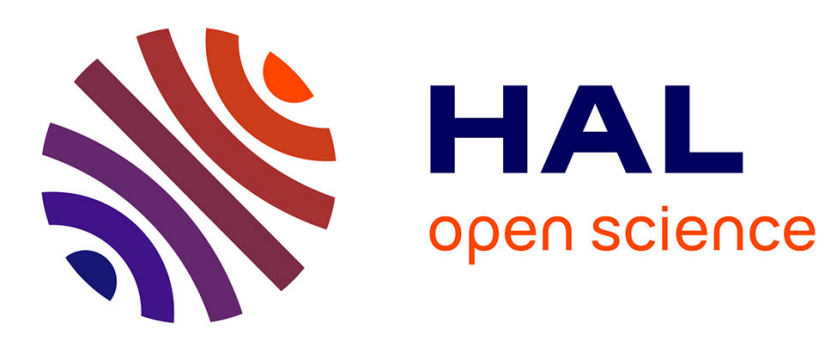

\title{
Estimation of the survival probabilities by adjusting a Cox model to the tail
}

\author{
Ion Grama, Jean Marie Tricot, Jean François Petiot
}

\section{To cite this version:}

Ion Grama, Jean Marie Tricot, Jean François Petiot. Estimation of the survival probabilities by adjusting a Cox model to the tail. C. R. Acad. Sci. Paris, Ser. I 349 (2011) 807-811, 2011, 349 (Ser. I), pp.807-811. 10.1016/j.crma.2011.06.011 . hal-00880127

\section{HAL Id: hal-00880127 https://hal.science/hal-00880127}

Submitted on 15 Nov 2013

HAL is a multi-disciplinary open access archive for the deposit and dissemination of scientific research documents, whether they are published or not. The documents may come from teaching and research institutions in France or abroad, or from public or private research centers.
L'archive ouverte pluridisciplinaire HAL, est destinée au dépôt et à la diffusion de documents scientifiques de niveau recherche, publiés ou non, émanant des établissements d'enseignement et de recherche français ou étrangers, des laboratoires publics ou privés. 


\title{
Estimation of the survival probabilities by adjusting a Cox model to the tail
}

\author{
Ion Grama ${ }^{\mathrm{a}}$, Jean-Marie Tricot ${ }^{\mathrm{a}}$, Jean-François Petiot ${ }^{\mathrm{a}}$ \\ ${ }^{a}$ Université de Bretagne Sud, Centre de recherche Yves Coppens, BP 573, 56017 VANNES, FRANCE \\ Received $* * * * *$; accepted after revision +++++ \\ Presented by
}

\begin{abstract}
Within the framework of a survival analysis model with censored life time data and an explanatory covariate, our goal is to predict the survival probability beyond the largest observed time. A Cox model with a constant underlying hazard function is proposed to adjust the tail of the life time distribution. Under some regularity conditions, we prove that the parameter estimators are convergent. To cite this article: I. Grama, J.M. Tricot, J.F. Petiot, C. R. Acad. Sci. Paris, Ser. I ??? (2010).

\section{Résumé}

Estimation des probabilités de survie par l'ajustement sur la queue d'un modèle de Cox. Dans le contexte d'un modèle d'analyse de survie avec données censurées et en présence d'une covariable explicative, on veut prédire la probabilité de survie au-delà de la plus grande observation. Un modèle de Cox dont la fonction de risque de base est constante, est ajusté sur la queue de la distribution de la durée de vie. Sous certaines conditions de régularité, on démontre la convergence des estimateurs des paramètres du modèle. Pour citer cet article : I. Grama, J.M. Tricot, J.F. Petiot, C. R. Acad. Sci. Paris, Ser. I??? (2010).
\end{abstract}

\section{Version française abrégée}

On utilise le modèle de Cox à hasard proportionnel $([6],[7])$ pour prédire la durée de vie à partir des temps d'observation censurés $t_{1}, \ldots, t_{n}$, lorsqu'elle est proche ou même dépasse max $\left\{t_{1}, \ldots, t_{n}\right\}$. Pour cela on propose d'ajuster la queue de la distribution de la durée de vie par un modèle de Cox. Cette approche est similaire à celle des références [2] et [3].

Email addresses: ion.grama@univ-ubs.fr (Ion Grama), jean-marie.tricot@univ-ubs.fr (Jean-Marie Tricot), jean-francois.petiot@univ-ubs.fr (Jean-François Petiot). 
On considère une variable aléatoire (v.a.) $X$ à valeur sur $\left[x_{0}, \infty\right), x_{0} \geq 0$, de fonction de répartition (f.r.) $F$, de densité $f_{F}$, de fonction de survie $S_{F}(x)=1-F(x), x \geq x_{0}$ et de fonction de risque $h_{F}=f_{F} / S_{F}$. Les temps de censure à droite, à valeur sur $\left[x_{0}, \infty\right)$, sont notés $C$. Soient $f_{C}$ et $F_{C}$ respectivement, les densité et f.r. de $C$. Soient $S_{C}(x)=1-F_{C}(x), x \geq x_{0}$ et $h_{C}=f_{C} / S_{C}$. On note $P_{F}(x, \delta), x \geq 0, \delta \in\{0,1\}$ la loi du vecteur $(T, \Delta)$, où $T=\min \{X, C\}$ et $\Delta=1(X \leq C)$. Soient $X_{1}, \ldots, X_{n}$ et $C_{1}, \ldots, C_{n}$ deux échantillons indépendants de $X$ et $C$ et soient $T_{i}=\min \left\{X_{i}, C_{i}\right\}$ et $\Delta_{i}=1\left(X_{i} \leq C_{i}\right)$. On note par $\mathbb{P}_{F}$ la loi des $Y_{i}=\left(T_{i}, \Delta_{i}\right), i=1, \ldots, n$ et par $\mathbb{E}_{F}$ l'espérance par rapport à $\mathbb{P}_{F}$. On suppose que $h_{F}(\cdot)=h(\cdot \mid z)$ dépend de la valeur $z$ d'une covariable $Z$ dans $\{0,1\}$. Par la suite on considérera une suite de réalisations $z_{1}, \ldots, z_{n}$ de $Z$ et les calculs se feront conditionnellement aux $Z_{i}=z_{i}$.

Notons $\lambda_{\beta, z} \equiv \lambda e^{\beta z}>0$. Pour $\lambda>0, \beta \in \mathbb{R}$ et $t>x_{0}$, notons $F_{\lambda, \beta, t}(x \mid z)$ la f.r. telle que :

$$
1-F_{\lambda, \beta, t}(x \mid z)=\left\{\begin{array}{cl}
S_{F}(x), & x \in\left[x_{0}, t\right], \\
S_{F}(t) \exp \left(-\lambda e^{\beta z}(x-t)\right), & x>t .
\end{array}\right.
$$

Soit $\widehat{n}_{z, t}=\sum_{T_{i} \geq t, Z_{i}=z} \Delta_{i}, z=0,1$. Les estimateurs locaux du maximum de la quasi-vraisemblance pour $\lambda_{\beta, 0}=\lambda$ et $\lambda_{\beta, 1}=\lambda e^{\beta}$ sont $: \widehat{\lambda}_{z, t}=\widehat{n}_{z, t} / \sum_{T_{i} \geq t, Z_{i}=z}\left(T_{i}-t\right), z=0,1($ avec $0 / 0=0)$.

Soit $\mathcal{K}\left(\lambda_{1}, \lambda\right)$ la divergence de Kullback-Leibler entre deux lois exponentielles de paramètres $\lambda_{1}>0$ et $\lambda>0$. Soit $\chi^{2}\left(P, P_{0}\right)=\int_{\mathbb{Y}} \frac{d P}{d P_{0}} d P-1$ l'entropie du $\chi^{2}$ entre les deux mesures de probabilité équivalentes $P$ et $P_{0}$. On dit que la queue de $F$ appartient au domaine d'attraction du modèle de Cox si il existe $\lambda>0$ et $\beta \in \mathbb{R}$ tels que $\lim _{t \rightarrow \infty} \chi^{2}\left(P_{F}, P_{F_{\lambda, \beta, t}}\right)=0$. Alors il existe une suite $\tau_{n} \in\left[x_{0}, \infty\right)$ telle que :

$$
\chi^{2}\left(P_{F}, P_{F_{\lambda, \beta, \tau_{n}}}\right)=O\left(\frac{\ln n}{n}\right) .
$$

Si $\xi_{n}$ et $\eta_{n}$ sont des v.a. non négatives, la notation $\xi_{n}=O_{\mathbb{P}_{F}}\left(\eta_{n}\right)$ signifie qu'il existe $c>0$ tel que $\mathbb{P}_{F}\left(\xi_{n} / \eta_{n} \leq c\right) \rightarrow 1$ lorsque $n \rightarrow \infty\left(\right.$ avec $\left.\frac{+\infty}{+\infty}=0\right)$.

Théorème 0.1 Si F appartient au domaine d'attraction du modèle de Cox et $\tau_{n}$ est une suite satisfaisant (0.1), alors $\mathcal{K}\left(\widehat{\lambda}_{z, \tau_{n}}, \lambda_{\beta, z}\right)=O_{\mathbb{P}_{F}}\left(\frac{\ln n}{\widehat{n}_{z, \tau_{n}}}\right)$, pour $z=0,1$.

Notons $q_{C, F}(t)=\int_{t}^{\infty} \frac{S_{C}(x)}{S_{C}(t)} \frac{f_{F}(x)}{S_{F}(t)} d x$ et $n_{z}=\sum_{i=1}^{n} 1\left(Z_{i}=z\right)$.

Théorème 0.2 Supposons qu'il existe deux constantes $q_{0}>0$ et $\kappa>0$ telles que $q_{C, F}(t) \geq q_{0}$, pour $t \geq$ $x_{0}$, et $\kappa \leq n_{z} / n \leq 1-\kappa$. Si F appartient au domaine d'attraction du modèle de Cox et $\tau_{n}$ est une suite satisfaisant (0.1) et $n S_{C}\left(\tau_{n}\right) S_{F}\left(\tau_{n}\right) \rightarrow \infty$ lorsque $n \rightarrow \infty$, alors $\mathbb{E}_{F} \widehat{n}_{z, \tau_{n}} \geq c_{0} \kappa n S_{C}\left(\tau_{n}\right) S_{F}\left(\tau_{n}\right)$, pour une constante $c_{0}>0, \mathbb{P}_{F}\left(\widehat{n}_{z, \tau_{n}} \geq \frac{1}{2} \mathbb{E}_{F} \widehat{n}_{z, \tau_{n}}\right) \rightarrow 1$ lorsque $n \rightarrow \infty$, et $\mathcal{K}\left(\widehat{\lambda}_{z, \tau_{n}}, \lambda_{\beta, z}\right)=O_{\mathbb{P}_{F}}\left(\frac{\ln n}{n S_{C}\left(\tau_{n}\right) S_{F}\left(\tau_{n}\right)}\right)$.

Soit $A>0, \lambda_{\max }>\lambda_{\min }>0, \beta_{\max }>\beta_{\min }$ des constantes. Dans le cas particulier où $F$ appartient à la classe $\mathcal{H}(\alpha)$ des fonctions telles que $h(x \mid z)=\lambda_{\beta, z}+r(x \mid z),|r(x \mid z)| \leq A \exp (-\alpha x)$, avec $\alpha>0$, $\lambda_{\min } \leq \lambda \leq \lambda_{\max }, \beta_{\min } \leq \beta \leq \beta_{\max }$, on peut donner la vitesse explicite en $n$. En effet, supposons la condition suivante concernant les censures :

$$
h_{C}(x)=\gamma+r_{C}(x),\left|r_{C}(x)\right| \leq M x^{-\mu}, \quad \mu>0, \gamma_{\min } \leq \gamma \leq \gamma_{\max },
$$

où $M>0, \gamma_{\max }>\gamma_{\min }>0$ sont des constantes. Notons $\lambda_{\beta}=\lambda \max \left\{1, e^{\beta}\right\}$.

Théorème 0.3 Soit $F \in \mathcal{H}(\alpha)$ et la condition (0.2) réalisée. Supposons qu'il existe deux constantes $q_{0}>0$ et $\kappa>0$ telles que $q_{C, F}(t) \geq q_{0}$, pour $t \geq x_{0}$, et $\kappa \leq n_{z} / n \leq 1-\kappa$. Alors, lorsque $z=0,1$, on a $\mathcal{K}\left(\widehat{\lambda}_{z, \tau_{n}}, \lambda_{\beta, z}\right)=O_{\mathbb{P}_{F}}\left(n^{-\frac{2 \alpha}{\lambda_{\beta}+\gamma+2 \alpha}} \ln n\right)$, où $\tau_{n}=\frac{\ln n}{\lambda_{\beta}+\gamma+2 \alpha}+O(1)$. 


\section{Introduction and main results}

The non parametric Cox proportional hazard model has become a common tool for predicting the time to event data in various applied fields (see for example [6], [7], [1] and the references therein, among many others). However it is well known that the estimated survival probabilities become very unstable when the life time is close to the right end of the observed data range. In the present paper we propose a new approach for estimating the survival probabilities from the censored failure time data $t_{1}, \ldots ., t_{n}$, when the life time is close to or even exceeds $\max \left\{t_{1}, \ldots, t_{n}\right\}$. Our idea consists in adjusting a Cox model to the tail of the unknown lifetime distribution and is similar to that used in [2] and [3].

Assume that a random variable (r.v.) $X$ with values in $\left[x_{0}, \infty\right), x_{0} \geq 0$, has the distribution function (d.f.) $F$, the density function $f_{F}$ and the survival function $S_{F}(x)=1-F(x), x \geq x_{0}$. By $h_{F}=f_{F} / S_{F}$ we denote its hazard function. Consider a right random censoring time $C$ with values in $\left[x_{0}, \infty\right)$ admitting the density $f_{C}$. Denote by $F_{C}$ its d.f. and let $S_{C}(x)=1-F_{C}(x), x \geq x_{0}$, and $h_{C}=f_{C} / S_{C}$. Let $P_{F}(x, \delta)$, $x \geq x_{0}, \delta \in\{0,1\}$ be the law of the random vector $(T, \Delta)$, where $T=\min \{X, C\}$ and $\Delta=1(X \leq C)$. The density of $P_{F}$ is given by $p_{F}(x, \delta)=f_{F}(x)^{\delta} S_{F}(x)^{1-\delta} f_{C}(x)^{1-\delta} S_{C}(x)^{\delta}, x \geq x_{0}, \delta \in\{0,1\}$.

Let $X_{1}, \ldots, X_{n}$ and $C_{1}, \ldots, C_{n}$ be two independent samples from $X$ and $C$ and let $T_{i}=\min \left\{X_{i}, C_{i}\right\}$ and $\Delta_{i}=1\left(X_{i} \leq C_{i}\right)$. Denote by $\mathbb{P}_{F}$ the joint law of the sample $Y_{i}=\left(T_{i}, \Delta_{i}\right), i=1, \ldots, n$ and by $\mathbb{E}_{F}$ the expectation w.r.t. $\mathbb{P}_{F}$ on $\mathbb{Y}^{n}$, where $\mathbb{Y}=\left[x_{0}, \infty\right) \times\{0,1\}$.

Assume that $S_{F}(x)=\exp \left(-\int_{x_{0}}^{x} h_{F}(v) d v\right)$, where $h_{F}(v)=h(v \mid z)$ is supposed to depend on the values $z$ of a covariate $Z$ with values in the set $\{0,1\}$. In the sequel we consider that we are given a sequence of realizations $z_{1}, \ldots, z_{n}$ of the covariate $Z$ and that all the reasonings are made conditionally w.r.t. these realizations.

Since the hazard function $h(\cdot \mid z)$ is not supposed to have a particular structure, the non-parametric estimator of the proposed model do not provide reliable estimation for the survival probabilities $S_{F}(x)$ for large values of $x$. In particular, when $x \geq \max \left\{t_{1}, \ldots, t_{n}\right\}$ for a given sample $t_{1}, \ldots, t_{n}$ of $T_{1}, \ldots, T_{n}$, these predictions are not meaningful. To ensure better predictive properties, we shall assume the Cox model (see for instance [6]) $h(x \mid z)=\lambda_{\beta, z} \equiv \lambda e^{\beta z}>0, x \geq t$, for some unknown threshold level $t \geq x_{0}$. For any $\lambda>0, \beta \in \mathbb{R}$ and $t \geq x_{0}$, denote by $F_{\lambda, \beta, t}(x \mid z)$ the distribution function defined by

$$
1-F_{\lambda, \beta, t}(x \mid z)=\left\{\begin{array}{cl}
S_{F}(x), & x \in\left[x_{0}, t\right] \\
S_{F}(t) \exp \left(-\lambda e^{\beta z}(x-t)\right), & x>t .
\end{array}\right.
$$

For any $t \geq x_{0}$ define quasi-likelihood by $L_{n}\left(F_{\lambda, \beta, t}\right)=\sum_{i=1}^{n} \ln p_{F_{\lambda, \beta, t}}\left(T_{i}, \Delta_{i}\right)$. For $z=0,1$, denote $\widehat{n}_{z, t}=\sum_{T_{i} \geq t, Z_{i}=z} \Delta_{i}$. Maximizing $L_{n}\left(F_{\lambda, \beta, t}\right)$ in $\lambda_{\beta, 0}=\lambda$ and in $\lambda_{\beta, 1}=\lambda e^{\beta}$ gives the local quasi likelihood estimators (with $0 / 0=0$ )

$$
\widehat{\lambda}_{z, t}=\frac{\widehat{n}_{z, t}}{\sum_{T_{i} \geq t, Z_{i}=z}\left(T_{i}-t\right)}, z=0,1 .
$$

Let $\mathcal{K}\left(\lambda_{1}, \lambda\right)$ be the Kullback-Leibler divergence between two exponential laws with parameters $\lambda_{1}>0$ and $\lambda>0$ respectively. It is easy to verify that $\mathcal{K}\left(\lambda_{1}, \lambda\right)=G\left(\frac{\lambda}{\lambda_{1}}-1\right)$, where $G(x)=x-\ln (x+1)$ for $x>-1$. By continuity we set $\mathcal{K}(0, \lambda)=+\infty$. Consider the $\chi^{2}$ entropy between two equivalent probability measures $P$ and $P_{0}: \chi^{2}\left(P, P_{0}\right)=\int_{\mathbb{Y}} \frac{d P}{d P_{0}} d P-1$. We say that the tail of the distribution function $F$ belongs to the domain of attraction of the Cox model if there exist $\lambda>0$ and $\beta \in \mathbb{R}$ such that $\lim _{t \rightarrow \infty} \chi^{2}\left(P_{F}, P_{F_{\lambda, \beta, t}}\right)=0$. It is easy to see that if the tail of the distribution function $F$ belongs to the domain of attraction of the Cox model then there is a sequence $\tau_{n}$ such that the following condition is satisfied 


$$
\chi^{2}\left(P_{F}, P_{F_{\lambda, \beta, \tau_{n}}}\right)=O\left(\frac{\ln n}{n}\right) .
$$

Now we are in position to formulate our main results. For non negative r.v.'s $\xi_{n}$ and $\eta_{n}$ the notation $\xi_{n}=O_{\mathbb{P}_{F}}\left(\eta_{n}\right)$ means that there exists $c>0$ such that $\mathbb{P}_{F}\left(\xi_{n} / \eta_{n} \leq c\right) \rightarrow 1$ as $n \rightarrow \infty$ (with $\left.\frac{+\infty}{+\infty}=0\right)$.

Theorem 1.1 If the distribution function $F$ belongs to the domain of attraction of the Cox model and $\tau_{n}$ is a sequence satisfying (1.1), then, for $z=0,1$,

$$
\mathcal{K}\left(\widehat{\lambda}_{z, \tau_{n}}, \lambda_{\beta, z}\right)=O_{\mathbb{P}_{F}}\left(\frac{\ln n}{\widehat{n}_{z, \tau_{n}}}\right) .
$$

Denote $q_{C, F}(t)=\int_{t}^{\infty} \frac{S_{C}(x)}{S_{C}(t)} \frac{f_{F}(x)}{S_{F}(t)} d x$. Remark that $q_{C, F}(t)$ is the proportion of the non-censored survival times among all survival times exceeding the threshold $t$. Denote $n_{z}=\sum_{i=1}^{n} 1\left(Z_{i}=z\right)$. Then $n_{z} / n$ is the proportion of observed times associated to the value $z$ of the covariate $Z$. The following theorem is a consequence of Theorem 1.1.

Theorem 1.2 Assume there exist constants $q_{0}>0$ and $\kappa>0$ such that $q_{C, F}(t) \geq q_{0}$, for $t \geq x_{0}$, and $\kappa \leq n_{z} / n \leq 1-\kappa$. If the distribution function $F$ belongs to the domain of attraction of the Cox model and the sequence $\tau_{n}$ is satisfying (1.1) and $n S_{C}\left(\tau_{n}\right) S_{F}\left(\tau_{n}\right) \rightarrow \infty$ as $n \rightarrow \infty$, then $\mathbb{E}_{F} \widehat{n}_{z, \tau_{n}} \geq$ $c_{0} \kappa n S_{C}\left(\tau_{n}\right) S_{F}\left(\tau_{n}\right)$, for some constant $c_{0}>0, \mathbb{P}_{F}\left(\widehat{n}_{z, \tau_{n}} \geq \frac{1}{2} \mathbb{E}_{F} \widehat{n}_{z, \tau_{n}}\right) \rightarrow 1$ as $n \rightarrow \infty$, and

$$
\mathcal{K}\left(\widehat{\lambda}_{z, \tau_{n}}, \lambda_{\beta, z}\right)=O_{\mathbb{P}_{F}}\left(\frac{\ln n}{n S_{C}\left(\tau_{n}\right) S_{F}\left(\tau_{n}\right)}\right) .
$$

Remark that the condition $q_{C, F}(t) \geq q_{0}$, for $t \geq x_{0}$, implies the identifiability of the model, for any fixed $t \geq x_{0}$.

\section{Explicit rate of convergence}

Let $A>0, \lambda_{\max }>\lambda_{\min }>0, \beta_{\max }>\beta_{\min }$ be some constants. We shall give the rate of convergence in the case when $F$ belongs to the class of distribution functions $\mathcal{H}(\alpha)$ with hazard function of the form $h(x \mid z)=\lambda_{\beta, z}+r(x \mid z),|r(x \mid z)| \leq A \exp (-\alpha x)$, where $\alpha>0, \lambda_{\min } \leq \lambda \leq \lambda_{\max }, \beta_{\min } \leq \beta \leq \beta_{\max }$. This class is similar to the class of functions studied in [4] and [5] for the extreme values models. To do so we shall impose the following condition on the censoring variable $C$ :

$$
h_{C}(x)=\gamma+r_{C}(x),\left|r_{C}(x)\right| \leq M x^{-\mu}, \quad \mu>0, \gamma_{\min } \leq \gamma \leq \gamma_{\max },
$$

where $M>0, \gamma_{\max }>\gamma_{\min }>0$ are some given constants. Denote $\lambda_{\beta}=\lambda \max \left\{1, e^{\beta}\right\}$.

Theorem 2.1 Assume $F \in \mathcal{H}(\alpha)$ and condition (2.1). Suppose that there exist constants $q_{0}>0$ and $\kappa>0$ such that $q_{C, F}(t) \geq q_{0}$, for $t \geq x_{0}$, and $\kappa \leq n_{z} / n \leq 1-\kappa$. Then, for $z=0,1$,

$$
\mathcal{K}\left(\widehat{\lambda}_{z, \tau_{n}}, \lambda_{\beta, z}\right)=O_{\mathbb{P}_{F}}\left(n^{-\frac{2 \alpha}{\lambda_{\beta}+\gamma+2 \alpha}} \ln n\right),
$$

where $\tau_{n}=\frac{\ln n}{\lambda_{\beta}+\gamma+2 \alpha}+O(1)$.

A consistent estimator of the parameter $\beta=\ln \frac{\lambda_{\beta, 1}}{\lambda_{\beta, 0}}$ is given by $\widehat{\beta}_{\tau_{n}}=\ln \frac{\widehat{\lambda}_{1, \tau_{n}}}{\widehat{\lambda}_{0, \tau_{n}}}$. A rate of convergence result for $\widehat{\beta}_{\tau_{n}}$ is straightforward. 


\section{Proofs of the main results}

Proposition 3.1 for any $\lambda, \lambda_{1}>0, \beta, \beta_{1} \in \mathbb{R}$ and any $x \geq 0$,

$$
\mathbb{P}_{F}\left(\sum_{i=1}^{n} \ln \frac{p_{F_{\lambda_{1}, \beta_{1}, t}}}{p_{F_{\lambda, \beta, t}}}\left(Y_{i}\right) \geq x+n \chi^{2}\left(P_{F}, P_{F_{\lambda, \beta, t}}\right)\right) \leq e^{-\frac{x}{2}} .
$$

The proof of the proposition is similar to that of Proposition 7.1 in [3] and therefore will be not detailed here.

Proposition 3.2 For any $\lambda>0, \beta \in \mathbb{R}, t \geq x_{0}$ and any $x \geq 0$ it holds

$$
\mathbb{P}_{F}\left(\widehat{n}_{z, t} \mathcal{K}\left(\widehat{\lambda}_{z, t}, \lambda_{\beta, z}\right) \geq x+n \chi^{2}\left(P_{F}, P_{F_{\lambda, \beta, t}}\right)+2 \ln n\right) \leq 2 e^{-\frac{x}{2}},
$$

where $z=0,1$.

The assertion of the Theorem 1.1 follows immediately from Proposition 3.2 if we set $x=2 \ln n$.

\subsection{Proof of Proposition 3.2.}

Note that

$$
\sum_{i=1}^{n} \ln \frac{p_{F_{\lambda_{1}, \beta_{1}, t}}}{p_{F_{\lambda, \beta, t}}}\left(Y_{i}\right)=\sum_{T_{i} \geq t} \Delta_{i} \ln \frac{\lambda_{1} e^{\beta_{1} z_{i}}}{\lambda e^{\beta z_{i}}}-\sum_{T_{i} \geq t}\left(\lambda_{1} e^{\beta_{1} z_{i}}-\lambda e^{\beta z_{i}}\right)\left(T_{i}-t\right) .
$$

Stratifying w.r.t. values of $Z_{i}$ we get

$$
\sum_{i=1}^{n} \ln \frac{p_{F_{\lambda_{1}, \beta_{1}, t}}}{p_{F_{\lambda, \beta, t}}}\left(Y_{i}\right)=\widehat{n}_{0, t} \Lambda_{0}\left(\lambda_{1}, \lambda_{\beta, 0}\right)+\widehat{n}_{1, t} \Lambda_{1}\left(\lambda_{1} e^{\beta_{1}}, \lambda_{\beta, 1}\right),
$$

where $\Lambda_{z}\left(\lambda^{\prime}, \lambda^{\prime \prime}\right)=\ln \frac{\lambda^{\prime}}{\lambda^{\prime \prime}}-\left(\lambda^{\prime}-\lambda^{\prime \prime}\right) \frac{1}{\lambda_{z, t}}, z=0,1$. Denote for brevity $y=x+n \chi^{2}\left(P_{F}, P_{F_{\lambda, \beta, t}}\right) \geq 0$ and $g(u, k)=\frac{\ln \frac{u}{\lambda_{\beta, z}}-\frac{y}{k}}{u-\lambda_{\beta, z}}$. Let $\lambda^{+}(k)=\arg \max _{u \geq \lambda_{\beta, z}} g(u, k)$ and $\lambda^{-}(k)=\arg \min _{u \leq \lambda_{\beta, z}} g(u, k)$. It is easy to see that

$$
\begin{aligned}
\left\{\widehat{n}_{z, t} \Lambda_{z}\left(\widehat{\lambda}_{z, t}, \lambda_{\beta, z}\right) \geq y, \widehat{\lambda}_{z, t} \geq \lambda_{\beta, z}\right\} & =\left\{g\left(\widehat{\lambda}_{z, t}, \widehat{n}_{z, t}\right) \geq \widehat{\lambda}_{z, t}, \widehat{\lambda}_{z, t} \geq \lambda_{\beta, z}\right\} \\
& \subset\left\{g\left(\lambda^{+}\left(\widehat{n}_{z, t}\right), \widehat{n}_{z, t}\right) \geq \widehat{\lambda}_{z, t}, \widehat{\lambda}_{z, t} \geq \lambda_{\beta, z}\right\} \\
& =\left\{\widehat{n}_{z, t} \Lambda_{z}\left(\lambda^{+}\left(\widehat{n}_{z, t}\right), \lambda_{\beta, z}\right) \geq y, \widehat{\lambda}_{z, t} \geq \lambda_{\beta, z}\right\} \\
& \subset\left\{\widehat{n}_{z, t} \Lambda_{z}\left(\lambda^{+}\left(\widehat{n}_{z, t}\right), \lambda_{\beta, z}\right) \geq y\right\} .
\end{aligned}
$$

In the same way we get

$$
\left\{\widehat{n}_{z, t} \Lambda_{z}\left(\widehat{\lambda}_{z, t}, \lambda_{\beta, z}\right) \geq y, \widehat{\lambda}_{z, t}<\lambda_{\beta, z}\right\} \subset\left\{\widehat{n}_{z, t} \Lambda_{z}\left(\lambda^{-}\left(\widehat{n}_{z, t}\right), \lambda_{\beta, z}\right) \geq y\right\} .
$$

These inclusions imply

$$
\begin{aligned}
\left\{\widehat{n}_{z, t} \mathcal{K}\left(\widehat{\lambda}_{z, t}, \lambda_{\beta, z}\right) \geq y\right\} & =\left\{\widehat{n}_{z, t} \Lambda_{z}\left(\widehat{\lambda}_{z, t}, \lambda_{\beta, z}\right) \geq y\right\} \\
& \subset\left\{\widehat{n}_{z, t} \Lambda_{z}\left(\lambda^{+}\left(\widehat{n}_{z, t}\right), \lambda_{\beta, z}\right) \geq y\right\} \cup\left\{\widehat{n}_{z, t} \Lambda_{z}\left(\lambda^{-}\left(\widehat{n}_{z, t}\right), \lambda_{\beta, z}\right) \geq y\right\} .
\end{aligned}
$$


From (3.1) we get

$$
\begin{aligned}
& \mathbb{P}_{F}\left(\widehat{n}_{z, t} \mathcal{K}\left(\widehat{\lambda}_{z, t}, \lambda_{\beta, z}\right) \geq y\right) \\
\leq & \mathbb{P}_{F}\left(\widehat{n}_{z, t} \Lambda_{z}\left(\lambda^{+}\left(\widehat{n}_{z, t}\right), \lambda_{\beta, z}\right) \geq y\right)+\mathbb{P}_{F}\left(\widehat{n}_{z, t} \Lambda_{z}\left(\lambda^{-}\left(\widehat{n}_{z, t}\right), \lambda_{\beta, z}\right) \geq y\right) \\
\leq & \sum_{k=1}^{n} \mathbb{P}_{F}\left(\widehat{n}_{z, t} \Lambda_{z}\left(\lambda^{+}(k), \lambda_{\beta, z}\right) \geq y\right)+\sum_{k=1}^{n} \mathbb{P}_{F}\left(\widehat{n}_{z, t} \Lambda_{z}\left(\lambda^{-}(k), \lambda_{\beta, z}\right) \geq y\right) .
\end{aligned}
$$

Suppose $z=0$. Choosing $\beta_{1}$ such that $\lambda_{1} e^{\beta_{1}}=\lambda e^{\beta}$, from Proposition 3.1, it follows

$$
\mathbb{P}_{F}\left(\widehat{n}_{0, t} \Lambda_{0}\left(\lambda_{1}, \lambda_{\beta, 0}\right) \geq y\right) \leq e^{-\frac{x}{2}},
$$

for any $\lambda_{1}>0$. Then, by (3.2) and (3.3),

$$
\mathbb{P}_{F}\left(\widehat{n}_{z, t} \mathcal{K}\left(\widehat{\lambda}_{0, t}, \lambda_{\beta, 0}\right) \geq y\right) \leq 2 n e^{-x / 2}=2 e^{-x / 2+\ln n} .
$$

The proposition is proved for $z=0$. The proof in the case $z=1$ is similar.

\subsection{Sketch of the proof of Theorem 2.1.}

Theorem 2.1 is a consequence of Theorem 1.1 if we show condition (1.1). Consider the distance $\rho_{t}\left(h_{1}, h_{2}\right)=\sup _{x>t}\left|h_{1}(x)-h_{2}(x)\right|$, where $h_{1}, h_{2}$ are two positive functions on $\left[x_{0}, \infty\right)$ and $t \geq x_{0}$. By Lemma 8.5 in [3],

$$
\chi^{2}\left(P_{F}, P_{F_{\lambda, \beta, \tau_{n}}}\right) \leq P_{F}\left(\ln \frac{d P_{F}}{d P_{F_{\lambda, \beta, \tau_{n}}}}\right)^{2} \exp \left(\left|\ln \frac{d P_{F}}{d P_{F_{\lambda, \beta, \tau_{n}}}}\right|\right) .
$$

Using the fact that $F \in \mathcal{H}(\alpha)$ and condition (2.1) we obtain

$$
\begin{aligned}
\chi^{2}\left(P_{F}, P_{F_{\lambda, \beta, \tau_{n}}}\right) & =O\left(S_{C}\left(\tau_{n}\right) S_{F}\left(\tau_{n}\right) \rho_{\tau_{n}}^{2}\right) \\
& =O\left(\exp \left(-\left(\gamma+\lambda_{\beta, z}+2 \alpha\right) \tau_{n}+\frac{A}{\alpha} e^{-\alpha \tau_{n}}+M \tau_{n}^{-\mu}\right)\right),
\end{aligned}
$$

where $\rho_{\tau_{n}}=\max _{z \in\{0,1\}} \rho_{\tau_{n}}\left(h(\cdot \mid z), \lambda e^{\beta z}\right)$. This implies that (1.1) holds with $\tau_{n}=\frac{\ln n}{\lambda_{\beta}+\gamma+2 \alpha}+O(1)$.

\section{References}

[1] J.-F. Dupuy, I. Grama, M. Mesbah, Asymptotic theory for the Cox model with missing time dependent covariate, Ann. of Statist. 34 (2006) 903-924.

[2] I. Grama, V. Spokoiny, Pareto approximation of the tail by local exponential modeling, Bulletin of Acad. of Sci. of Moldova 1 (53) (2007) 1-22.

[3] I. Grama, V. Spokoiny, Statistics of extremes by oracle estimation, Ann. Statist. 36 (2008) 1619-1648.

[4] P. Hall, A.H. Welsh, Best attainable rates of convergence for estimates of parameters of regular variation, Ann. Statist. 12 (1984) 1079-1084.

[5] P. Hall, A.H. Welsh, Adaptive estimates of regular variation, Ann. Statist. 13 (1985) 331-341.

[6] J.P. Klein, M.L. Moeschberger, Survival Analysis: Techniques for Censored and Truncated Data, Springer, 2003.

[7] J.D. Kalbfleisch, R.L. Prentice, The Statistical Analysis of Failure Time Data, Wiley, 2002. 